\title{
Pattern-Segmented Heart Rate Variability Analysis During Fetal Maturation
}

\author{
Alexander Schmidt ${ }^{1}$, Dirk Hoyer ${ }^{1}$, Uwe Schneider ${ }^{2}$ \\ ${ }^{1}$ Biomagnetic Center, Hans Berger Department of Neurology, Jena University Hospital, Germany \\ ${ }^{2}$ Department of Obstetrics, Jena University Hospital, Germany
}

\begin{abstract}
Background: Established fetal maturation diagnostics evaluates heart rate patterns (HRP) such as baseline, variability, decelerations (DC) and accelerations (AC). Fetal heart rate variability ( $f H R V$ ) parameters provide valuable additional information. Their dependence on those patterns is crucial but not sufficiently explored. Objective of the present work is the comparison of the maturational age prediction using fHRV parameters with respect to those patterns.

Methods: We analyzed 555 recordings, each one lasting 30 minutes, of normal fetuses, from 19 weeks of gestation (WGA) onward. We applied a patternsegmented fHRV analysis of linear and nonlinear parameters under consideration of the fetal behavioral states to evaluate the WGA prediction accuracy, based on linear regression models that were tested using a repeated cross validation scheme.

Results/Conclusion: $f H R V$ parameters calculated under exclusion of DCs, show a significantly improved age dependency compared to the standard method, especially in the early weeks of the second half pregnancy. This aspect may improve the early sensitive identification of maturation disorders of the fetus.
\end{abstract}

\section{Introduction}

Monitoring the fetal heart rate (fHR) and the assessment of fHRV plays a central role in the pre- and perinatal clinical surveillance and management of the fetus. The standard bedside method applied today is cardiotocography (CTG), which is based on ultrasound and delivers a cardiac tachogram for which specific measures of fHRV have been developed. Computerized fHRV rate analysis was introduced in Oxford by Dawes and Redman in 1982. In 1989, a system to analyze HRV was created based on a database of 8000 fetal heart rate recordings. fHRV parameters like short-term variation (STV) and long term variation (LTV) are now established in obstetric practice for surveillance of fetal well-being together with the Dawes-Redman criteria for normality.

This approach does, however, not lend itself to the analysis of HRV according to the standards developed for routine electrocardiogram (ECG) with higher sampling rate and identifying individual heart beats, equivalent to the magnetocardiogram (MCG) applied in the fetus. fHRV in time, frequency and complexity domains were previously mainly been analysed over the entire time series without selecting particular pattern segments.

The traditional assessment of fetal well-being is based on the visual inspection by a trained gynecologist and the FIGO guidelines for CTG classification. Similar to the Dawes-Redman criteria fHR is analyzed mainly with respect to baseline, variability, DCs and ACs. These aspects influence the calculated fHRV parameters. ACs are defined as an increase in FHR above the baseline for longer than 15 seconds and have a maximum excursion above the baseline of greater than 15 beats per minute (bpm). A DC is defined as fHR decrease below baseline for longer than 15 seconds and has a maximum excursion below the baseline of greater than $15 \mathrm{bpm}$. The fetal HRP displayed in CTG depends on gestational age, fetal activity and a variety of other factors, but classification of recordings primarily aimed on distinguishing the healthy from the distressed fetuses rather than precisely assessing maturation [1].

The Fetal Autonomic Brain Age Score (fABAS) is based on a system biology approach that applies universal principles of evolution and self-organization to the ontogenetic functional development of the fetus. It can be understood as a proxy for the neural integration of the developing organism. fABAS estimates the gestational age by means of a multivariate linear model based on fHRV indices. The coefficients of fABAS resulted from a learning set of normal developing fetuses between 19 and 40 weeks of gestation (WGA) [2].

Very little is known about how fHRV parameters differ with respect to different fHRP and calculation window length, and to what extent the statistical properties of these pattern segmented fHRV parameters allow a more precise estimation of the fetal maturation compared to the established standard fHRV analysis.

The aims of the present study are I) to compare the predicted fABAS values based on the different segmented HRV parameters and different window lengths.

II) to see to what extent the established univariate standard HRV parameters are influenced by the fHRP in the course of pregnancy and under consideration of the fetal behavioral states.

In that context, segmentation of the measured HR under consideration of fetal behavioral state as well as of 
the particular exclusion of ACs, DCs and baseline correction may provide information about different aspects of autonomic modulations in more detail.

\section{Methods}

Data was obtained from the study database at the Biomagnetic Center, Department of Neurology and from the Department of Obstetrics, both at the Jena University Hospital. We investigated in total 555 recordings from 165 mothers with singleton normal maturating pregnancies during non-stress situations aged between 19 and 40 WGA. Approval was obtained from the local Ethics Committee of the Friedrich Schiller University. All subjects gave their written consent to participate in the study.

All measurements were taken in a magnetically shielded room. The fMCG recordings were performed during daytime over a period of 30 min sampling rate $1024 \mathrm{~Hz}$, using the 195 channel vector-magnetograph ARGOS200 (ATB, Chieti, Italy). The pregnant women were positioned supine or with a slight twist to either side to prevent compression of the inferior vena cava by the pregnant uterus. The Dewar was positioned as close as possible but without contact above the fetal heart, determined by sonographic localization.

From the entire $30 \mathrm{~min}$ recordings we analyzed sections of active sleep (2F) and quiet sleep (1F) with a minimum duration of 10 minutes. These states were selected by visual inspection of HRP printout and consensus decision by three independent obstetricians according to the standard criteria, which are extended to gestational ages prior to 32 WGA [3]. Generally, a single episode per state and dataset was considered. Episodes of quiet awakeness (3F) and active awakeness (4F) were not considered separately due to their rare occurrence.

The heart beats were detected using an independent component-based strategy [4] and normal-to-normal (NN) beat interval series were constructed after visual inspection for artifacts and ectopic beats.

In addition to the original fHR (orig), periods without DCs (oD), segments with neither DCs nor ACs (basal) and the baseline corrected fHR (baseline) were analyzed in the state sections. The basal activity was only considered in sections of quiet sleep, due to the fact that longer episodes of basal activity are only extractable there (Figure 1).

The methodology of the here proposed MCG based segmented HRV analysis is different from the traditional FIGO guidelines. In the present context, the definitions of AC and DC were modified according to the following rules due to the higher temporal resolution of the fMCG and the fact that before 32 WGA, their amplitude and duration may be lower:

- An AC is defined as an increase in the heartrate for more than 5 seconds with a deviation $\geq 10 \mathrm{bpm}$.
- A DC is defined as a decrease below the baseline for more than 5 seconds and a deviation $\geq 20 \mathrm{bpm}$.

In order to detect ACs and DCs a baseline needs to be determined. There is no gold standard for fitting a baseline, and it is normally assigned by means of visual assessment, but occasionally, it may be difficult or even impossible to guess where the baseline should be. The baseline estimation follows mainly the original DawesRedman baseline fitting procedure [5] with some minor changes, which can be summarized in the following steps:

a) Partitioning of the data into no overlapping 5 minute segments in order to react to strong baseline drifts

b) Creation of the frequency distribution of the epochby-epoch average pulse intervals and scanning the distribution from right to left until a peak value is identified that satisfies some specific conditions

c) Delimitation of the baseline by not considering values that are not within the range of $\pm 5 \mathrm{bpm}$ from the calculated peak value

d) Combining the single 5 minute baseline segments and smooth them with a 101 Heartbeat mean window

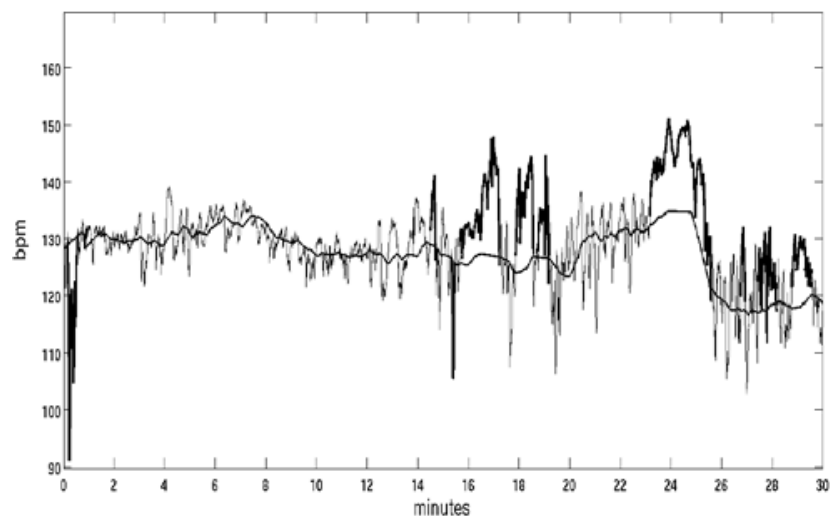

Figure 1: A 30-minute tachogram (0-12 min 1F, 12-30 2F) in which DC and AC were marked thick. The solid black line shows the estimated baseline.

The ACs and DCs were marked by threshold detection, removed from the FHR signal and the remaining segments are concatenated accordingly to the considered pattern segmented fHRV analysis. Figure 1 shows an example file in which the segmentation is illustrated. Within the first 12 minutes the fetus is in $1 \mathrm{~F}$ state followed by 18 minutes $2 \mathrm{~F}$.

In order to compensate the effect of different data lengths of the state segments, all parameters are determined by means of a sliding window. The following window lengths were considered: 5,6,7,8,9,10 minutes with an overlap of 2 minutes for all HRV parameters. The parameters gMSE(1-4), LZC and of the frequency domain represent exceptions to the above description since they use already a sliding window during their calculation procedure. 
This kind of pattern segmented window HRV calculation was performed for all significantly age related HRV parameters of fABAS and the most frequently used linear HRV parameters from time and frequency domains, as well as some of the nonlinear HRV parameters. On each of the four differently segmented NN interval series, 23 different HRV parameters were calculated.

In order to ensure a normal distribution of the HRV parameters different transformations functions (sqrt/log) were tried and the resulting distributions were tested for normality via Shapiro Wilk test and the visual inspection of the QQ-plot. An overview of the calculated parameters and the used transformation functions can be seen in table 1. For calculation details please see Schmidt et al. [6].

The signal analysis was performed using MatLab2014a and the statistical analysis using $\mathrm{R}$ version 3.3.2. The influence of the different segmented fHRV parameters on WGA prediction was examined on the basis of univariate linear regression models. The goodness of fit parameter SE (standard error) is estimated via a 10 -fold cross validation with 3 repeats. The SE's from the single folds are tested via Wilcoxon signed rank sum test for significant differences. The significance level was set to 0.05 . The same test procedure and settings were used for the comparison of the different multivariate fABAS models.

\section{Results}

Episodes of active sleep could be found in 449 of the 555 recorded datasets (80.9\%) and fetal quiescence in 181 traces $(32.6 \%)$. Due to the small number of $1 \mathrm{~F}$ files and for reasons of space, only the results of the $2 \mathrm{~F}$ analyses are shown.

Figure 2 shows the standard error of the three different fABAS models together with their standard deviation for different window sizes from 5 up to 10 minutes in the $2 \mathrm{~F}$ state. The scores are based on their corresponding pattern segmented fHRV parameters. Overall, the differences in accuracy for the three different fABAS models (orig, oD, baseline) at each window length are significant at the transitions from 5-6, 6-7 and 7-8 minutes.

When comparing the models within the different window lengths, the fABAS-oD model is always significantly worse (by $<0.1$ WGA). The fABASbaseline model can assert itself as a significantly better model from the 7 minute window onwards. For the further presentation of the univariate results, we limited ourselves to the results of the 5-min segments.

Table 1 shows the SE of the univariate regression models of the considered fHRV parameters. There are only few significant differences between the original and the baseline corrected models. The changes are in the range of magnitude around the $0.01 \mathrm{SE}$.

The majority of the significant differences are only observed in fHRV parameters calculated without DCs. With the exception of the non-linear fHRV parameters, meanHR, Amp and the pnn5 all other parameters show improvements of the SE values by 0.2 to 0.4 . The highest improvement can be seen by the frequency parameters closely followed by rmssd. For skew and $\alpha_{2}$ the removing of DCs had a negative effect.

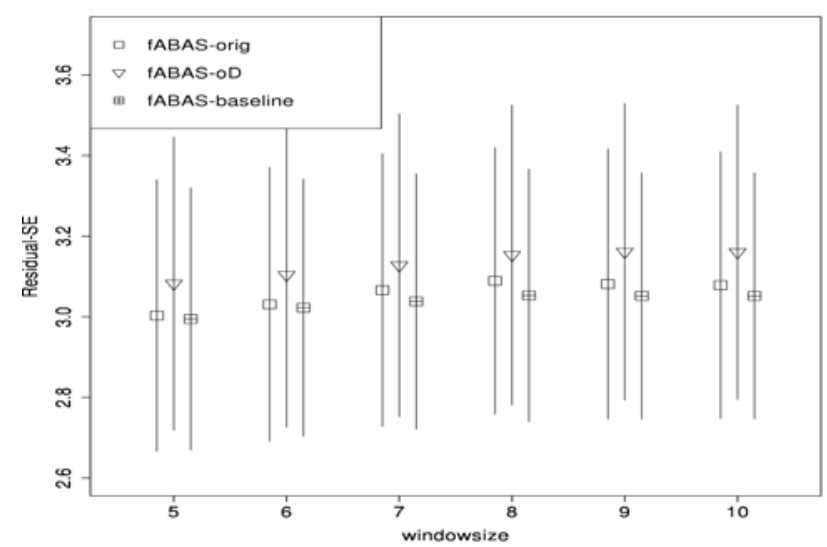

Figure 2: SE \pm standard deviation of the three fABAS scores for different window sizes from 5 up to 10 minutes in the 2F state

From the fABAS related HRV parameters Amp, skewness (skew) and pnn5 provided strong univariate age predictors. The 2 strongest predictors are Amp and pnn5 with a SE of 3.51 and 3.59. They are followed by the P0V, a parameter from the family of symbolic dynamics, which captures fast signal changes. fABAS-orig predicts the maturational age significantly better than Amp by an amount of $0.5 \mathrm{SE}$.

\section{Discussion/Conclusion}

In this work we could primarly show a systematic effect on single fHRV parameters of the time and frequency domain by excluding DCs.

In the multivariate case using fABAS, the removal of DCs significantly worsened the results. In comparison to the univariate parameters, the multivariate estimation of maturational age remained very stable among the considered HRP and window lengths with an accuracy of 3 WGA. For the shorter window lengths some minor accuracy changes could be found as well as some significant accuracy improvements through a baseline correction before calculating HRV parameters. However, these changes are only on the magnitude of 0.01-0.02 weeks. These minimal changes are not relevant for clinical routine in the pre- and perinatal clinical surveillance and management of the fetus.

In the univariate case, in contrast to the multivariate results, the exclusion of DCs clearly improved the prediction accuracy. DCs occur primarily in the early phase of pregnancy and become progressively less as the 
fetus matures. Accordingly, the most pronounced effect can be observed in the early weeks of the pregnancy period studied here. In the FIGO guidelines DCs play a central role in interpretation of CTG and the classification of abnormal fHR traces. For those reasons, it is advisable to treat the DCs separately before calculating fHRV parameters, especially when considering younger pregnancy periods. By contrast, the non-linear fHRV parameters show only minor changes by removing DCs. This may be due to their internal processing method. The parameters quantify the predictability of a signal and are independent of the signal's variation range. The few significant accuracy changes within the baseline corrected parameters are again in a negligible range of magnitude.

The segmentation according to behavioral states classification is the result of a consensus decision of three experts based on HRP that furthermore slightly change with maturation age between 19 to 40 WGA. This classification is not always unambiguous, but it reflects the heterogeneity of recordings and the state of the art.

Traditional HRV indices reflect predominantly vagal activity during $1 \mathrm{~F}$ and sympathetic activity during $2 \mathrm{~F}$. The presented pattern-segmentation methodology may allow a more accurate assessment of the complex sympathetic and parasympathetic modulations. This capability may allow a more sophisticated identification of maturation disorders of the fetus. This methodology is intended to contribute to further exploration and validation with regard to the early identification of developmental disorders using independent data sets in multicenter studies.

\section{Acknowledgements}

AS, DH, US were supported by German Research Foundation DFG (Ho 1634/15-12, Schn 775/7-1, Ho 1634/12-2, Schn 775/2-3).

\section{References}

[1] (Federation Internationale de Gynecologie et d'Obstetrics), Maternity Guidelines, Federal Gazette, Institute for Quality and Efficiency in Health Care, Germany, 2011.

[2] D. Hoyer et al., "Fetal functional brain age assessed from universal developmental indices obtained from neurovegetative activity patterns," PLoS One, vol. 8, no. 9, p. e74431, 2013.

[3] J. G. Nijhuis, H. F. Prechtl, C. B. Martin, Jr., and R. S. Bots, "Are there behavioural states in the human fetus?," (in eng), Early Hum Dev, vol. 6, no. 2, pp. 177-95, Apr 1982.

[4] A. Schmidt, U. Schneider, O. W. Witte, E. Schleussner, and D. Hoyer, "Developing fetal motor-
Table 1: The SE \pm standard deviation of the univariate regression models. For parameters marked with *, no transformation could be found to achieve a normal distribution. All other parameters are normally distributed. Thick printed numbers mark significant differences in comparison to the orig parameter based on a Wilcoxon signed rank test.

\begin{tabular}{|c|c|c|c|c|}
\hline \multirow{2}{*}{\multicolumn{2}{|c|}{\begin{tabular}{|c|} 
HRV \\
parameters \\
\end{tabular}}} & \multicolumn{3}{|c|}{ SE } \\
\hline & & orig & oD & baseline \\
\hline \multirow{6}{*}{ 声 } & meanHR & $4.31 \pm 0.27$ & $4.30 \pm 0.27$ & $4.30 \pm 0.27$ \\
\hline & $\lg (\operatorname{sdnn})$ & $4.20 \pm 0.28$ & $3.82 \pm 0.30$ & $4.19 \pm 0.29$ \\
\hline & $\lg (\mathrm{rmssd})$ & $4.41 \pm 0.22$ & $3.98 \pm 0.29$ & $4.41 \pm 0.22$ \\
\hline & sqrt(pnn5) & $3.65 \pm 0.29$ & $3.59 \pm 0.29$ & $3.64 \pm 0.30$ \\
\hline & skew* & $3.53 \pm 0.33$ & $3.77 \pm 0.35$ & $3.47 \pm 0.32$ \\
\hline & $\log ($ Amp) & $3.54 \pm 0.33$ & $3.51 \pm 0.34$ & $3.54 \pm 0.34$ \\
\hline \multirow[b]{2}{*}{$\frac{a}{\text { pa }}$} & sqrt(STV) & $3.94 \pm 0.31$ & $3.73 \pm 0.27$ & $3.96 \pm 0.31$ \\
\hline & sqrt(LTV) & $4.17 \pm 0.28$ & $3.78 \pm 0.28$ & $4.20 \pm 0.28$ \\
\hline \multirow{5}{*}{ 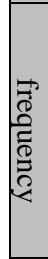 } & $\log ($ Total $)$ & $4.45 \pm 0.25$ & $3.81 \pm 0.31$ & $4.45 \pm 0.25$ \\
\hline & $\log (\mathrm{VLF})$ & $4.42 \pm 0.26$ & $3.79 \pm 0.32$ & $4.42 \pm 0.26$ \\
\hline & $\log (\mathrm{LF})$ & $4.48 \pm 0.24$ & $3.96 \pm 0.28$ & $4.48 \pm 0.24$ \\
\hline & $\log (\mathrm{IMF})$ & $4.45 \pm 0.25$ & $4.25 \pm 0.23$ & $4.45 \pm 0.25$ \\
\hline & $\log (\mathrm{HF})$ & $4.42 \pm 0.23$ & $4.03 \pm 0.29$ & $4.42 \pm 0.23$ \\
\hline \multirow{10}{*}{ 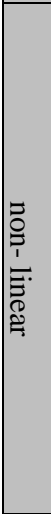 } & gMSE1 & $4.46 \pm 0.22$ & $4.47 \pm 0.22$ & $4.45 \pm 0.22$ \\
\hline & gMSE2 & $4.26 \pm 0.27$ & $4.28 \pm 0.27$ & $4.27 \pm 0.27$ \\
\hline & gMSE3 & $4.16 \pm 0.29$ & $4.15 \pm 0.28$ & $4.16 \pm 0.29$ \\
\hline & gMSE4 & $4.17 \pm 0.29$ & $4.16 \pm 0.28$ & $4.18 \pm 0.28$ \\
\hline & $\mathrm{POV}^{*}$ & $3.67 \pm 0.31$ & $3.65 \pm 0.3$ & $3.67 \pm 0.31$ \\
\hline & P1V* & $3.76 \pm 0.30$ & $3.74 \pm 0.29$ & $3.76 \pm 0.31$ \\
\hline & $\mathrm{P}^{2} \mathrm{~V}^{*}$ & $3.70 \pm 0.34$ & $3.70 \pm 0.33$ & $3.71 \pm 0.34$ \\
\hline & $\alpha_{1}$ & $4.48 \pm 0.24$ & $4.45 \pm 0.26$ & $4.48 \pm 0.24$ \\
\hline & $\alpha_{2}$ & $4.35 \pm 0.25$ & $4.47 \pm 0.24$ & $4.34 \pm 0.25$ \\
\hline & LZC & $4.47 \pm 0.24$ & $4.47 \pm 0.25$ & $4.48 \pm 0.23$ \\
\hline
\end{tabular}

cardiovascular coordination analysed from multichannel magnetocardiography," Physiol. Meas., vol. 35, pp. 1943-1959, 2014.

[5] J. Pardey, M. Moulden, and C. W. Redman, "A computer system for the numerical analysis of nonstress tests," Am J Obstet Gynecol, vol. 186, no. 5, pp. 1095-103, May 2002.

[6] A. Schmidt et al., "Universal characteristics of evolution and development are inherent in fetal autonomic brain maturation," Autonomic Neuroscience - Basic \& Clinical, vol. 212, pp. 32-41, Jul 2018.

Address for correspondence:

M.Sc. Alexander Schmidt

Hans Berger Department of Neurology-Biomagnetic Center Jena University Hospital

Am Klinikum 1

D 07747 Jena, Germany

Alexander.Schmidt2@med.uni-jena.de 